\title{
Learning in organisations
}

In his essay The Face of Miranda, ${ }^{1}$ the eminent palaeontologist Stephen Jay Gould explains how scientific theory can be overturned by new facts. A notion he proposed that planetary surfaces were a consequence of size and nothing else worked adequately for our local planets of Mars, Mercury, Earth and its moon. But more distant images from the unmanned Voyager spaceship revoked his theory. ${ }^{1}$ When Voyager approached Miranda, the smallest satellite of Uranus, a NASA scientist declared excitedly:

'It's just mind-boggling ... Miranda is what you get if you imagine taking all the bizarre geological features in the solar system and putting them all into one object.'

Gould concluded a size hypothesis by itself was not strong enough 'to overwhelm other influences' and yield confident predictions. He wrote in his essay that theories about the surfaces of planets should be descriptive rather than predictive and experimental. Planets are complex objects of history, not at all like uniform billiard balls.

Coincidentally, at the same time as this correction, a small team based at Lancaster University was also overturning some basic ideas. By the mid-1970s Lancaster's Department of Systems had established a reputation within the industrial sector as consultants in stock control and distribution. Working on behalf of numerous clients, the department's small teams found statistical forecasting invaluable when improving the flow of items within or beyond a factory. However, Peter Checkland, a new appointment at Lancaster, directed the teams into quite different situations where applying advanced mathematics proved inappropriate. An unusual distinguishing feature of these new organisational issues was ambiguity: clients seemed reluctant or unable to say what kind of problems they had.

An approach to research named Soft
Systems Methodology (SSM) ${ }^{2}$ evolved that helped clarify such hazy situations by carefully preparing diagrams to sharpen discussion. Brief English phrases in these systems diagrams replaced mathematics as the researchers' tool for investigation and so made the approach more likely to foster participation. In the best investigations people taking part eagerly dropped assumptions that their organisations were systems and began to use that word to refer to diagrams constructed for the sake of their own purposeful research. Instead of the social world holding systems, systems were deployed to look into the problematical social world.

To illustrate Professor Checkland's methodology consider, for example, a health centre attempting to promote a healthy lifestyle among local university students by giving tips on diet and exercise. Most of the centre's staff believe this kind of intervention will alleviate the students' future health problems. A systems diagram that illustrates promoting lifestyle tips as a purposeful set of activities is shown below:

i. find out about students' future health problems, which diet and exercise can alleviate;

\section{$\downarrow$}

ii. define hints on diet and exercise to alleviate the future health problems; $\downarrow$

iii.decide how to deliver hints to students; $\downarrow$

iv. prepare and deliver lifestyle hints to students.

In this admittedly sparse example, the numbers $i$ to iv given to the four phrases are shorthand for easy reference to each activity. The arrows represent a flow of information/resources between activities. If agreed as relevant to participants' concerns, this diagram is contrasted against local knowledge of the status quo. Each of the system's activities can structure questions about current arrangements. Does this activity exist in some form? How is the activity carried out? What is the measure of performance? What would count as an improvement? Should the diagram fail to generate much serious parley, new systems can be sketched and proposed in a continuous process of learning and debate; the lifestyle of local students might be just one of several underlying issues at the health centre.

Voyager's mission was to return images of unique distant planets, so complementing our scientific knowledge of mankind's small part in the vast universe. However SSM, though also a system to assist learning, never supplies images of complex social relationships. SSM diagrams are merely inexpensive 'tools' which can be set alongside heterogeneous human organisations far more intricate than any extra-terrestrial world we know. Whether expressing conservative or radical views, the diagrams should prove pertinent to enlivening discussion within a particular culture at a particular time.

\section{Neil Richardson}

\section{REFERENCES}

1. Gould SJ. The face of Miranda. In: Bully for Brontosaurus: Reflections In Natural History. London: Hutchinson Radius, 1991: 489-498.

2. Checkland P, Poulter J. Learning for action: a short definitive account of soft systems methodology, and its use for practitioners, teachers and students. Chichester, UK: John Wiley \& Sons Ltd, 2006.

\section{DOI: 10.3399/bjgp09X472746}

